\title{
LA LITTÉRATURE COMME TÉMOIN
}

Témoignages et littérature d'après Auschwitz, par Fransiska Louwagie, Brill, Amsterdam 2020, 377 pp., €127, ISBN: 978-90-04-42609-2 (hardback); 978-90-04-43068-6 (e-book).

https://doi.org/10.19195/0557-2665.68.19

Témoignages et littérature d'après Auschwitz présente un aperçu de la création littéraire qui, faisant fi des interdictions contre l'esthétisation d'une tragédie dite sans précédent et inexprimable ${ }^{1}$, depuis la guerre continue à attester des souffrances des victimes de la barbarie nazie. Plus spécifiquement, Fransiska Louwagie se donne pour objectif d'interroger les enjeux éthiques et esthétiques posés par les ouvrages étudiés. Elle considère ceux derniers dans une perspective à la fois comparative et transversale, c'est-à-dire en lien avec l'évolution de la mémoire de la guerre en France et avec d'autres écrits testimoniaux. Ainsi, l'auteure poursuit-elle sa réflexion soutenue sur la période de Vichy, dont témoignent ses nombreux ouvrages consacrés à Gérard Wajcman, Jorge Semprun, Piotr Rawicz et Henri Raczymow. Plus récemment, Louwagie s'est distinguée avec son travail novateur sur l'ego-histoire'2.

Comme tout spécialiste en littérature concentrationnaire, Louwagie se heurte au problème de l'unité de son corpus. La plupart des études existantes assurent celle-ci en sélectionnant les auteurs en fonction de leur appartenance linguistique, de leur catégorie de déporté (racial/politique), de leur sexe, ou de la distance générationnelle qui les sépare de la guerre. Au lieu d'imposer à son corpus une homogénéité potentiellement inféconde ou même inopérante, Louwagie se penche

${ }^{1}$ Ici, il convient de citer la formulation célèbre de Theodor W. Adorno : « écrire un poème après Auschwitz est barbare » et celle d'Elie Wiesel qui a proclamé qu'un roman sur Treblinka soit n'était pas un roman, soit n'était pas sur Treblinka (T.W. Adorno, « Kulturkritik und Gesellschaft », [dans :] Prismen: Kulturritik und Gesellschaft, Deutschen Taschenbuch Verlag, Munich 1963, p. 26 — cité par K. Hoffmann, « Poetry after Auschwitz: Adorno's Dictum », German Life and Letters 52.2, avril 2005, p. 182 ; E. Wiesel, « The Holocaust as Literary Inspiration », [dans :] E. Wiesel, L.S. Dawidowicz, D. Rabinowicz et R. McAfee Brown (dir.), Dimensions of the Holocaust. Lectures at Northwestern University, Northwestern University Press, Evanston, IL, 1977, p. 7).

${ }^{2}$ M. Bragança et F. Louwagie (dir.), Ego-histories of France and the Second World War. Writing Vichy, Palgrave MacMillan, Basingstoke 2018. 
à la fois sur les auteurs-survivants et sur ceux qui, n'ayant pas vécu la déportation eux-mêmes, ont un rapport post-mémoriel à celle-ci. De même, elle porte attention aux écrivains juifs et non-juifs, aux auteurs francophones ainsi qu'à ceux qui, comme Imre Kertész, s'expriment dans d'autres langues, et, finalement, à diverses modalités génériques. Il n'empêche que l'auteure est consciente de l'hétérogénéité de son corpus ; c'est précisément cette diversité qui a inspiré l'étiquette de « la littérature d'après Auschwitz», où Auschwitz est utilisé métonymiquement pour désigner " une rupture culturelle », plutôt qu'un camp de concentration précis (p. 2). Ce qui réunit alors les œuvres abordées par Louwagie est leur farouche volonté de transmettre l'expérience de la violence nazie ou, autrement dit, leur adhérence au « pacte testimonial ». Analogique au «pacte autobiographique », tel qu'il a été défini par Philippe Leujene, ce dernier se manifeste par des constatations « je le jure », «j’y étais » ou « je me souviens » (p. 16).

Témoignages et littérature d'après Auschwitz se divise en trois parties. La partie théorique (qui fait office d'introduction à l'ouvrage) offre une esquisse de l'évolution de la littérature testimoniale et des débats qui entourent celle-ci. Ces débats portent sur la valeur historique du témoignage littéraire, laquelle a été mise en question par Fragments (1995) de Binjamin Wilkomirski ${ }^{3}$; sur son caractère inévitablement fictionnel ; sur l'identité du témoin (le bourreau peut-il se poser en témoin historique fiable ?) ; sur la tension entre l'histoire et la mémoire à «l'ère du témoin $»^{4}$; et, finalement, sur la notion de « témoin de témoin » à l'époque où les survivants se font de plus en plus rares. Ensuite, Louwagie introduit la terminologie utilisée dans son étude, y compris le terme clé « œuvre-témoignage » (p. 4). Emprunté à Claude Mouchard ${ }^{5}$, celui-ci se réfère à un récit qui, rédigé sous l'effet des destructions massives et organisées du $\mathrm{XX}^{\mathrm{e}}$ siècle, s'engage dans le pacte testimonial, tout en s'inscrivant dans le champ littéraire.

Ce sont les " œuvres-témoignages » de cinq écrivains qui font l'objet de l'analyse dans la première partie analytique. Chapitre 1 s'attarde sur le questionnement de l'humanisme, qui est au cœur du témoignage d'Robert Antelme de son emprisonnement à Buchenwald en tant que déporté politique. La faible réception de L'Espèce humain (1947) est symptôme de la domination de l'imaginaire populaire par le résistantialisme gaullien, selon lequel les Français auraient unanimement résisté contre les nazis, et qui a marginalisé l'expérience des déportés, tout en étouffant la spécificité de la mémoire juive ${ }^{6}$. Chapitre 2 se tourne vers André Schwartz-Bart, un écrivain quelque peu oublié aujourd'hui, qui, à l'encontre d'Antelme, de Jorge Semprun ou de Piotr Rawicz, a échappé à la déportation. Récompensé par le Prix Goncourt, son roman Le Dernier de Justes (1958) conçoit

3 Fragments s'est avéré une fiction élaborée et son auteur un imposteur.

4 A. Wieviorka, L'Ère du témoin, Hachette, Paris 2002.

${ }^{5}$ C. Mouchard, Qui si je criais...? Ouvres-témoignages dans les tourments du XXe siècle, Laurence Teper, Paris 2007.

${ }^{6}$ Le récit n'a connu succès qu'en 1957, quand il a été réédité chez Gallimard. 
Auschwitz comme l'accomplissement de l'antisémitisme européen. Le chapitre mentionne aussi les écrits que Schwartz-Bart a consacrés au racisme et à l'esclavage, ce qui, à la lumière des théories récentes, aurait fait de son écriture emblème de « la mémoire palimpseste » ou de « la mémoire multidirectionnelle » ${ }^{7}$. Le chapitre suivant est dédié à Rawicz, écrivain juif-polonais, qui a survécu à la Shoah en cachant son identité. Son roman Le Sang du ciel (1961) a polarisé la critique par, d'un côté, son rejet de la pudeur associée à l'écriture concentrationnaire à travers la place importante qu'il accorde à la sexualité, et, de l'autre, sa représentation des victimes juives en complices de leurs bourreaux.

Dans la deuxième partie analytique, Louwagie dirige son attention, d'abord, vers « la génération liminale ». Mieux connue comme « la génération 1.5 », celleci comprend ceux qui, comme Georges Perec ou Raymond Federman, étaient enfants pendant la guerre ${ }^{8}$. Contrairement à l'œuvre perequienne, qui a été amplement étudiée, celle de Federman reste méconnue en France, malgré l'intérêt y porté dans le monde anglophone ${ }^{9}$. Ayant échappé à la rafle du Vel' d'Hiv grâce à sa mère, qui l'a poussé au dernier moment dans un débarras, Federman s'est établi aux États-Unis, où il s'est adonné à l'étude de l'auteur franco-irlandais, Samuel Becket, tout en créant sa propre œuvre littéraire bilingue et expérimentale. Sur le plan thématique, les ouvrages non-linéaires et métafictionnels de l'auteur reviennent souvent sur sa survie quasi-miraculeuse et sur la perte de sa famille.

Si les écrits des auteurs de la deuxième génération analysés dans la suite du livre sont en majorité plus conventionnels que la surfiction de Federman, le roman de Gérard Wajcman en fait exception. Notamment, L'Interdit (1986) se compose surtout des notes infrapaginales assez énigmatiques, tandis que le texte est «perdu, effacé ou absent» (p. 283). Wajcman lui-même explique la mise en forme de son roman par l'exclusion de la langue yiddish qu'il a subie enfant. La deuxième partie analytique s'achève par une analyse de l'œuvre de Michel Kichka, fils d'Henri Kichka, témoin infatigable de son expérience concentrationnaire. Né en Belgique, mais vivant en Israël, Kichka est connu pour son travail d'illustrateur et de caricaturiste satirique. Inspiré par Maus d'Art Spiegelman, son roman graphique Deuxième génération. Ce que je n'ai pas dit à mon père (2012) se focalise, contrairement à Maus qui s'intéresse surtout aux parents de l'auteur, sur l'expérience des enfants des survivants.

7 M. Silverman, Palimpsestic Memory. The Holocaust and Colonialism in French and Francophone Fiction and Film, Berghahn Books, New York 2013 ; M. Rothberg, Multidirectional Memory. Remembering the Holocaust in the Age of Decolonisation, Stanford University Press, Stanford, CA, 2009.

${ }^{8}$ S. Rubin Suleiman, «The 1.5 Generation : Thinking about Child Survivors and the Holocaust », American Imago 59.3, automne 2002, pp. 277-295.

9 J.R. Di Leo (dir.), Federman's Fictions. Innovation, Theory, Holocaust, SUNY Press, New York 2011. L'ouvrage collectif comporte des essais par Dan Stone, Susan Suleiman et Brian McHale. 
En somme, le livre de Louwagie est une tentative opportune et importante de combler la pénurie d'études de la littérature de la Shoah dans la langue française. D'ailleurs, en choisissant les auteurs qui « viennent d'horizons différents, [qui] ont été sujets à des expériences variées, [qui] se réfèrent à divers cadres interprétatifs et s'expriment à travers un large éventail de formes littéraires et de langues » (p. 4), l'auteure nous invite à contempler la création des écrivains qui se côtoient rarement dans des ouvrages critiques. Son livre offre une analyse claire et lucide, qui s'appuie sur une érudition exceptionnelle en ce qui concerne la période de Vichy. Il est dommage, pourtant, que Louwagie - surtout en tant que femme - ne fasse références aux écrivains de son propre sexe qu'en passant. J'aurais aimé lire des chapitres sur Sarah Kofman ou sur la romancière judéo-polonaise de la langue française, Anna Langfus, dont le roman Les Bagages de sable (1962) a obtenu le Prix Goncourt et dont l'œuvre très riche n'a pas encore reçu la considération qu'elle mérite.

Helena Duffy

ORCID: 0000-0002-6047-9952

Université de Wrocław / Turun Yliopisto helena.duffy@uwr.edu.pl /helena.duffy@utu.fi

\title{
UN PANORAMA DES DICTIONNAIRES ESPAGNOLS
}

\author{
Niezwykte losy słowników. Historia leksykografi hiszpańskiej \\ [L'exceptionnel destin des dictionnaires. Histoire de la lexicographie espagnole], \\ par Ewa Stala, Wydawnictwo Uniwersytetu Jagiellońskiego, Kraków 2020, \\ 208 pp., ca $€ 8$ (paperback), ISBN: 978-83-233-4897-9. \\ https://doi.org/10.19195/0557-2665.68.20
}

Le titre du dernier ouvrage d'Ewa Stala - lexicologue, lexicographe, métalexicographe et historienne de la langue espagnole ${ }^{1}$, professeure à l'Université Jagellonne de Cracovie — ne dévoile que partiellement son contenu. On pourrait croire, par exemple, qu'il s'agit uniquement de la lexicographie monolingue et même, seulement de la lexicographie monolingue générale. Or, le champ d'inves-

${ }^{1}$ Voir notamment : M. Filipowicz-Rudek, E. Stala, Kieszonkowy stownik hiszpańsko-polski, polsko-hiszpański, Wydawnictwo Zielona Sowa, Kraków 2004 (2 éd. 2008) ; E. Stala, Los dobletes etimológicos en español (1611-1739), Wydawnictwo Uniwersytetu Jagiellońskiego, Kraków 2012 ; E. Stala, El léxico español en el "Waaren-Lexicon in zwölf Sprachen" de Ph. A. Nemnich, Peter Lang, Frankfurt am Main 2015. 